\title{
Interplay between Stride Velocity, Stride Frequency and Stride Length in Human Gait Transition
}

\author{
Doppelhammer $\mathbf{N}$, Baumgartner $\mathbf{W}$ and Vereshchaga $\mathbf{Y}^{*}$ \\ Institute of Biomedical Mechatronics, Johannes Kepler University Linz, Altenbergerstr. 69, 4040 Linz, Austria
}

\begin{abstract}
This paper presents the results of studying human gait transition outside the laboratory setting on a treadmill by means of wearable sensor technology. Combined inertial measurement and pressure sensor units embedded in footwear were employed to analyze the movement of the lower limbs during walk-run and run-walk transitions. Experimental data from 20 subjects was used to study three parameters - stride velocity, stride length and stride frequency. We focused on seven strides centered around the transition stride, that is, the stride in-between walking and running that has only a single floating phase. Three subjects underwent additional testing on a treadmill to capture the differences in kinematics between the two environments. The stride frequency varied least with both a subject's individual transition behavior and the environment. The former can be concluded from the standard deviations evaluated for each stride, which were lower for stride frequency than for velocity and length. The latter was derived by comparing the results from within and outside the laboratory: Stride frequency shifted similarly in both cases, mainly within 2-4 strides, to the attractor of the new gait. Velocity profiles differed, with acceleration being more uniform and much lower on the treadmill. Stride lengths were inversely proportional: In the walk-run transition, when the belt of the treadmill was sped up, stride lengths decreased. In the run-walk transition, when the belt was slowed down, stride lengths increased. This phenomenon is attributable mainly to the dominant nature of the stride frequency, which forces changes in stride length in order to fulfill the velocity constraint. A non-laboratory environment lacks such a constraint, thus giving rise to free transition behavior. Stride frequency, being easy to measure, is well suited to analyzing and defining gait in a practical context.
\end{abstract}

Keywords: Biomechanics; Gait transition; Gait analysis; Anthropometric parameters; IMU sensor technology

\section{Introduction}

Development of assisting technologies which predict human intention and enable biomechanical analysis of movement for safety or rehabilitation purposes is of interest in many applications, including research into active exoskeletal robotic systems. Here, the aim is, for instance, to predict the user's intended movements to enable a seamless transition between walking and running [1]. A prerequisite of tackling this task is gaining a profound understanding of how humans perform gait transition in order to identify the principal influencing factors [2].

Human locomotion is considered to take two primary forms: walking and running. Walking is a form of locomotion defined by double-support phases, in which both feet are on the ground at the same time. Running is a form of locomotion that has only single-support phases: When humans run, the feet are never in contact with the ground simultaneously. The phases in which both feet are temporarily airborne are called floating phases. Normally, gait is changed at speeds around 2.0 $\mathrm{m} / \mathrm{s}[3,4]$. The mechanism that triggers gait transition is multi-facetted and depends on individual trained behaviors, body composition, physical constitution and psychological state. An extensive theoretical model that provides a detailed biomechanical description of how transition is performed has yet to be developed - an onerous task due to the numerous influential factors.

Conclusions drawn from human gait transition investigated on a treadmill do not automatically apply outside the laboratory, since many kinematic, physiological and perceptual differences exist. This could be, for example, the absence of optical flow or differences in cognitive-affective mechanisms, such as when the subject is intimidated by unforeseeable accelerations. The most crucial difference is probably that, on a treadmill, the velocity of a subject's lower limbs is constrained by the speed of a moving belt. Unsurprisingly, studies have reported remarkable differences in gait transition between the two environments. Both the spontaneously chosen acceleration and the preferred transition speed were considerably higher outside the laboratory than in the treadmill case $[5,6]$.

Previous studies have focused mainly on investigating the (transition) speed and less on other parameters that influence transition behavior [3,7-9]. Though a recently published investigation in laboratory conditions on a treadmill proposes that the transition happens as the stride frequency of the walking attractor shifts to the frequency of the running attractor [10]. Stride frequency and stride length are two further interesting parameters, because they are closely related to stride velocity via

$$
v_{s}=f_{s} l_{s}
$$

This relationship is valid independent of the environment. On a treadmill, stride velocity is constrained, which means that the user cannot adapt frequency and length independently. Outside the laboratory setting, velocity can be adjusted freely. Thus, the question arises of how stride parameters behave during transition and how their interplay is influenced by environmental changes.

In this paper we investigated human transition based on the stride

${ }^{*}$ Corresponding author: Vereshchaga $\mathrm{Y}$, Institute of Biomedical Mechatronics, Johannes Kepler University Linz, Altenbergerstr. 69, 4040 Linz, Austria, Tel: +43 7322468 4806; Fax: +43 7322468 24801; E-mail: yana.vereshchaga@jku.at

Received June 11, 2018; Accepted June 22, 2018; Published June 29, 2018

Citation: Doppelhammer N, Baumgartner W, Vereshchaga Y (2018) Interplay between Stride Velocity, Stride Frequency and Stride Length in Human Gait Transition. J Bioanal Biomed 10: 74-79. doi:10.4172/1948-593X.1000209

Copyright: (C) 2018 Doppelhammer N, et al. This is an open-access article distributed under the terms of the Creative Commons Attribution License, which permits unrestricted use, distribution, and reproduction in any medium, provided the original author and source are credited. 
parameters $v_{s}, f_{s}$ and $l_{s}$. For measurements outside the laboratory we developed a wearable measurement system that combines inertial measurement units (IMUs) with pressure sensors and collected data from 20 volunteers. We studied seven strides centered around the transition stride (TS) and investigated both the walk-run transition (WRT) and the run-walk transition (RWT). Three subjects were additionally tested on a treadmill to capture behavioral differences in the two environments.

We found that stride frequency is largely unaffected by (1) the individual transition behavior and (2) the environment. Velocity and length, however, show significant differences. Therefore stride frequency is the property of choice to analyze human gait shift in practical contexts. It adapts rapidly and robustly to changes in gait and can be measured easily.

\section{Methods}

\section{Participants}

Twenty healthy volunteers $(26 \pm 3.24$ years; $61.3 \pm 11.17 \mathrm{~kg}$; $174.7 \pm 8.31 \mathrm{~cm}$ ), 10 male and 10 female, participated in this study (Table 1). None of them reported injuries or any other pathology that would prevent them from walking or running.

\section{Procedures}

Experiments outside the laboratory: The participants were first tested under non-laboratory conditions. They were instructed to cover a distance of $77.6 \mathrm{~m}$ in a straight line on flat, asphalted ground. Participants were asked to start walking and increase their speed up to a point at which they felt comfortable to change gait. After transition, the running speed was to be kept at a moderate level before changing back to the walking gait. The total distance was to be covered $1 / 3$ walking, $1 / 3$ running and $1 / 3$ walking. 10 measurements per participant were recorded. Measurements were rejected if,

(i) A faulty measurement signal was received (e.g., insufficient pressure on the pressure sensors during one or more strides).

(ii) A stumbling stride occurred inside the transition region.

(iii) Regions of uniform gait were too small/large. From the pool of valid measurements, the first five were chosen for further analysis.

Treadmill experiments: Participants 5, 6 and 11 (Table 1) were also tested on a treadmill. Each participant walked on a standard treadmill, starting at a speed of $1.12 \mathrm{~m} / \mathrm{s}$ and accelerating continuously by $0.056 \mathrm{~m} / \mathrm{s}^{2}$. At a belt speed of $2.78 \mathrm{~m} / \mathrm{s}$, the speed was maintained for $10 \mathrm{sec}$. Then the belt was decelerated at $-0.056 \mathrm{~m} / \mathrm{s}^{2}$ down to the initial speed of $1.12 \mathrm{~m} / \mathrm{s}$ and kept at this level for another $10 \mathrm{sec}$.

In both environments, WRT and RWT were clearly separated from each other, with a considerably long running gait in between. We assume that during this time, gait parameters are fully adapted and therefore an effect of a non-randomized order of WRT and RWT can be excluded.

\section{Instruments}

Measurements outside the laboratory: We developed a wearable measurement system, as shown in Figure 1, to acquire measurement data in a non-laboratory setting. 3D acceleration and rotational speed were recorded with an MPU6050 (InvenSens Inc., USA) 3D inertial measurement unit (IMU). For maximum comfort, the IMU was placed beneath the shoe's insole under the foot's horizontal arc at slight medial

\begin{tabular}{|c|c|c|c|c|c|c|c|c|c|c|}
\hline ID & Age & SSPW & Mass [kg] & Height [cm] & LM [cm] & TH [cm] & $\mathrm{TL}[\mathrm{cm}]$ & TrH $[\mathrm{cm}]$ & $\mathrm{SH}[\mathrm{cm}]$ & $\mathrm{FL}[\mathrm{cm}]$ \\
\hline $1 \mathrm{M}$ & 26 & 3 & 70.0 & 177.2 & 7.0 & 47.5 & 44.0 & 91.8 & 91.0 & 26.0 \\
\hline $2 \mathrm{M}$ & 28 & 3 & 69.5 & 179.1 & 7.2 & 49.0 & 49.0 & 93.0 & 91.5 & 28.0 \\
\hline $3 \mathrm{M}$ & 30 & 0 & 86.8 & 174.5 & 7.1 & 49.0 & 45.0 & 85.5 & 95.9 & 26.0 \\
\hline $4 \mathrm{M}$ & 28 & 2 & 84.6 & 179.4 & 7.1 & 51.3 & 46.0 & 100.5 & 92.7 & 26.0 \\
\hline $5 \mathrm{M}$ & 16 & 0 & 61.1 & 184.6 & 7.9 & 53.9 & 45.0 & 99.0 & 95.0 & 26.5 \\
\hline $6 \mathrm{M}$ & 26 & 2 & 72.0 & 178.4 & 7.8 & 46.5 & 41.0 & 100.6 & 95.0 & 27.5 \\
\hline $7 \mathrm{M}$ & 24 & 3 & 68.5 & 170.4 & 6.8 & 44.8 & 38.0 & 89.4 & 89.0 & 25.5 \\
\hline $8 \mathrm{M}$ & 28 & 3 & 81.0 & 181.2 & 8.0 & 48.5 & 40.0 & 97.9 & 95.0 & 27.5 \\
\hline $9 \mathrm{M}$ & 26 & 3 & 79.0 & 189.0 & 7.4 & 53.5 & 49.0 & 107.2 & 93.0 & 26.5 \\
\hline $10 \mathrm{M}$ & 26 & 1 & 74.0 & 182.0 & 7.0 & 48.1 & 42.5 & 98.0 & 95.0 & 25.5 \\
\hline $11 \mathrm{~F}$ & 26 & 3.5 & 58.5 & 171.5 & 7.3 & 47.0 & 39.5 & 95.6 & 91.3 & 24.2 \\
\hline $12 \mathrm{~F}$ & 26 & 1.5 & 61.5 & 155.8 & 7.1 & 42.0 & 35.0 & 83.6 & 87.3 & 23.0 \\
\hline $13 \mathrm{~F}$ & 25 & 2 & 51.0 & 174.8 & 7.3 & 46.9 & 40.0 & 98.5 & 91.0 & 24.5 \\
\hline $14 \mathrm{~F}$ & 23 & 0 & 55.5 & 173.5 & 7.1 & 46.3 & 38.0 & 92.0 & 93.5 & 23.0 \\
\hline $15 \mathrm{~F}$ & 26 & 2 & 59.0 & 160.3 & 6.5 & 42.5 & 35.0 & 86.8 & 86.0 & 23.5 \\
\hline $16 \mathrm{~F}$ & 25 & 2 & 52.0 & 168.4 & 7.6 & 45.1 & 40.0 & 98.5 & 87.0 & 23.5 \\
\hline $17 \mathrm{~F}$ & 20 & 3 & 58.0 & 170.9 & 7.5 & 45.3 & 38.0 & 97.3 & 90.0 & 24.0 \\
\hline $18 \mathrm{~F}$ & 24 & 3 & 58.5 & 163.9 & 7.2 & 44.0 & 37.5 & 93.4 & 86.0 & 23.5 \\
\hline $19 \mathrm{~F}$ & 23 & 4 & 52.2 & 164.5 & 6.8 & 41.6 & 36.0 & 90.4 & 87.0 & 24.0 \\
\hline $20 \mathrm{~F}$ & 20 & 1 & 57.1 & 176.0 & 6.8 & 48.7 & 41.0 & 95.0 & 93.0 & 22.5 \\
\hline Mean & 26 & 2 & 61.3 & 174.7 & 7.2 & 47.0 & 40.0 & 95.3 & 91.4 & 25.0 \\
\hline SD & 3.2 & 1.2 & 11.2 & 8.3 & 0.4 & 3.4 & 4.2 & 5.8 & 3.3 & 1.7 \\
\hline
\end{tabular}

Note: SSPW=Sport Sessions Per Week; M=males; F=Females; LM=Lateral Malleolus Height; TiH=Tibial Height; TrH=Trochanteric Height; TL=Thigh Length; SH = Sitting Height; FL=Feet Length.

Table 1: Data from the 20 subjects (10 males, 10 females) tested in a non-laboratory setting. Subjects $5,6 \& 11$ also underwent testing on a treadmill. The table includes characteristic body measurements to allow the relation between transition behavior and body composition to be studied; however, this task was outside the scope of this work. 


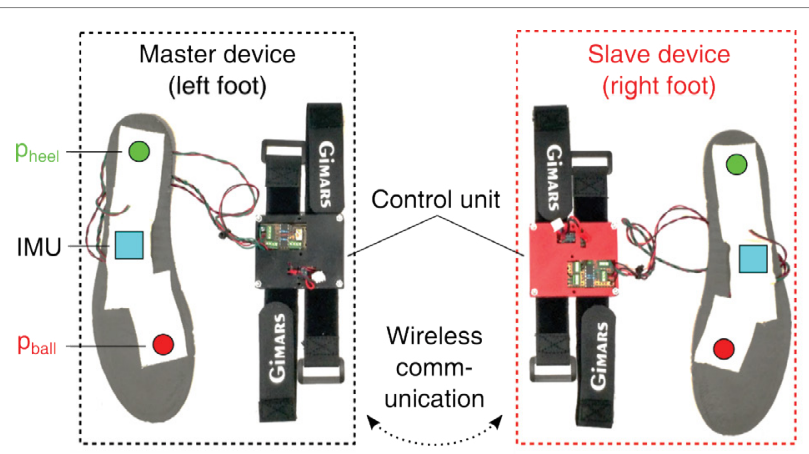

Figure 1: Measurement system for investigating walk-run and run-walk transitions outside the laboratory. Insoles were equipped with sensors to measure stride parameters and detect gait events. $\mathrm{P}_{\text {heel }}$ and $\mathrm{P}_{\text {ball }}$ denote the pressure sensors on heel and ball respectively. The IMU is placed under the longitudinal foot arch inside a special housing for maximum comfort. The control unit, responsible for data acquisition, storage, power supply and timesynchronization between master and slave device, was fixed laterally to the lower shank. Whenever a measurement is manually started on the master device, measurement begins synchronously on the slave device, as both devices are connected wirelessly via Bluetooth.

position. To detect the floating phases, Flexiforce A301 (Tekscan, USA) pressure sensors were placed under heel and ball. Left and right feet were instrumented identically. All subjects confirmed that the wearable sensors did not hinder their movement.

Data of each sub device was acquired by means of an Arduino Nano at a rate of $265 \mathrm{~Hz}$ and stored on a microSD card for further processing. A lithium-ion polymer battery was used for power supply and ensured autonomous operation. Bluetooth modules established wireless communication between the control units on both feet. Whenever a measurement was triggered on the master device on the left foot, a start command was sent to the right side that forced simultaneous measurement to begin on the slave device. All components other than the sensors were combined in a separate housing fixed to the lower shank with hook and loop fasteners.

Treadmill measurements: For the treadmill experiments, a video-based motion-capture system was used to track the movement of the lower limbs. The movement of the foot was recorded with a standard DSLR camera (Canon D700) at a frame rate of $50 \mathrm{fps}$. A marker was placed laterally on the right shoe at the bottom midfoot position. The camera was oriented perpendicular to the sagittal body plane and placed at a distance of $3.2 \mathrm{~m}$, capturing the entire motion of the feet. It was set to maximum zoom to reduce the effect of radial lens distortion.

Software: Raw measurement data was postprocessed with functions developed in house using MATLAB 2016b (The MathWorks, Inc., Natick, Massachusetts, USA), which are available from the authors on request. Foot trajectories were obtained from the videos by means of the open-source motion analysis software Kinovea ${ }^{\odot}$.

\section{Data analysis}

Non-laboratory case: The MPU6050 measures 3D acceleration and rotational speed in a body-fixed frame. Acceleration in the world frame is calculated with the help of rotation matrices. Mathematically, the transformation can be written as

$$
a_{w}=A_{w b} a_{\text {grav }, b}-g_{w}
$$

where $A_{w b}$ is a $3 \times 3$ matrix that translates $3 \mathrm{D}$ acceleration from the body frame (index b) to the world frame (index w); a direction cosine matrix approach is used to successively process $A_{w b}$ from gyroscopic data. $a_{\text {grav }, b}$ is the acceleration according to earth's gravitational field measured in the body frame. $a_{w}$ and $g_{w}=\left[\begin{array}{lll}0 & 0 & 9.81 \mathrm{~m} / \mathrm{s}\end{array}\right]^{T}$ are the vectors of linear acceleration and gravity in the world frame, respectively. By time integration of the world frame acceleration, we obtain the velocity

$$
v_{w}(t)=\int a_{w}(t) d t
$$

double integration yields the distance

$$
s_{w}(t)=\int v_{w}(t) d t=\int a_{w}(t) d t
$$

Drift is a well-known problem when integrating noisy and biased sensor data from an IMU, which can be counteracted by utilizing the periodicity of the human gait: The velocity and the orientation of the body frame are reset when the foot lies flat at midstance. The midstance event is reliably detected as the first intersection of the normalized heeland ball-pressure functions after heel strike. Data-processing is paused until midstance because the accelerometer signal is heavily affected by artifacts due to the striking heel, and foot movement is negligible during this time period, as illustrated in Figure 2.

A similar approach has previously been employed in several studies [11-13], often referring to the stride-wise reset of the integration error as zero-velocity update (ZUPT). For IMUs in pedestrian navigation, this measure drastically decreases the measurement error and is indispensable if no other sophisticated filter technique, such as an extended Kalman filter, is applied. Stride length, stride frequency and stride velocity are obtained by

$$
\begin{gathered}
l_{s}=\int_{T_{s}}^{T_{s+1}} v_{w, x} d t \\
f_{s}=\frac{1}{\Delta T_{s}}, \\
v_{s}=l_{s} f_{s}
\end{gathered}
$$

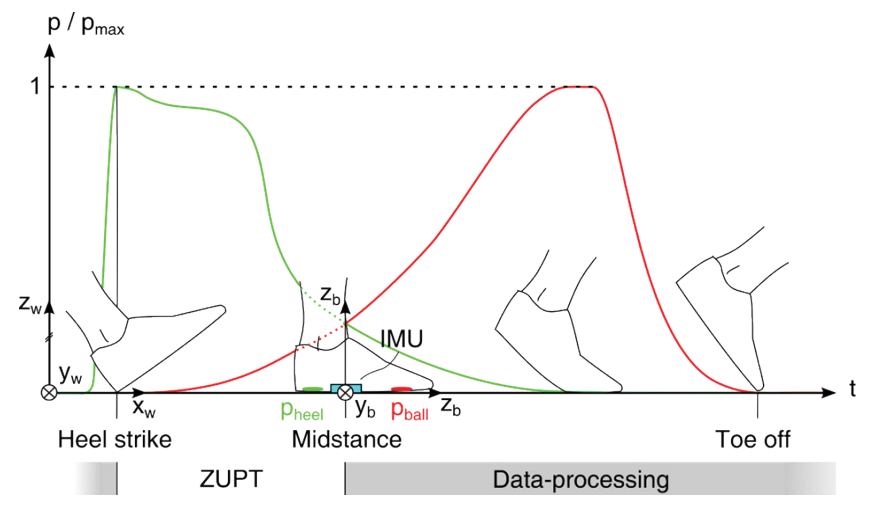

Figure 2: Zero-velocity update (ZUPT) for minimizing the integration error when using IMUs for pedestrian velocity and distance measurements. Shown is the stance phase of the human gait ranging from heel strike to toe off. When the foot lies flat at midstance, the integration error and the orientation of the local frame are reset. Thus, the integration interval is divided into numerous small sections, which reduces the error dramatically when integrating biased and noisy sensor data. Midstance is defined as the intersection of the ball $\left(p_{\text {ball }}\right)$ and heel $\left(\mathrm{p}_{\text {heel }}\right)$ pressure functions. 
Here, $\Delta T_{s}=T_{s+1}-T_{s}$ is the time difference between two successive heel-strike events. Note that stride velocities and lengths in the $y$ - and $\mathrm{z}$ - directions vanish due to the boundary condition (straight, planar locomotion along the global $\mathrm{x}$-axis). This holds because we assume that the initial orientation of the IMU is aligned with that of the world frame.

Having obtained the stride parameters for all twenty subjects, we calculated the mean stride parameters and mean standard deviations. First, we computed mean and standard deviation for the five measurements per subject via

$$
\bar{p}_{i}=\frac{1}{N_{m p s}} \sum_{j=1}^{N_{m p s}} p_{i j}
$$

and

$$
\sigma_{i}=\sqrt{\frac{\sum_{j=1}^{N_{m p s}}\left(\bar{p}_{i}-p_{i j}\right)}{N_{m p s}-1}}
$$

Where $\bar{p}_{i}$ represents the $i$-th subject's mean parameter value $(p$ either $v_{s}, f_{s}$ or $l_{s}$ ) and $\sigma_{i}$ its standard deviation. $\mathrm{N}_{m p s}=5$ is the number of measurements per subject and $p_{i j}$ denotes the $\mathrm{j}$-th measurement of the i-th subject. Summing all $\bar{p}_{i}$ and $\sigma_{i}$ and dividing by the number of subjects $\mathrm{N}_{s}$ yields respectively the mean parameter values and mean standard deviation of the whole group,

$$
\bar{p}=\frac{1}{N_{s}} \sum_{i=1}^{N_{s}} p_{i}
$$

and

$$
\bar{\sigma}=\frac{1}{N_{s}} \sum_{i=1}^{N_{s}} \sigma_{i}
$$

Equations (8-11) must be applied to each of the seven strides within the transition region in both WRT and RWT.

Treadmill case: After tracking the marker positions with Kinovea, we processed the position data further in MATLAB. First, data was upsampled from $50 \mathrm{~Hz}$ to $256 \mathrm{~Hz}$ by linear interpolation to obtain the same sample rate as in the non-laboratory setting. The velocity was then obtained by time-derivation of the position vector. Translation from the local belt-fixed frame to the global frame was achieved by a coordinate transformation of the form

$$
v_{w}(t)=v_{b}(t)-v_{b e l t}(t)
$$

Where $v_{w}$ and $v_{b}$ are vectors in the world and the body frame, respectively. $v_{\text {belt }}(t)=\left[v_{\text {belt }, x}(t), 0,0\right]^{T}$ is the adjusted velocity profile of the moving belt. Stride velocity, stride frequency and stride length are, again, obtained by Equations (5-7). A drawback of video-based measurement is occlusion, where one foot sometimes obscures the other. As this drastically decreases the accuracy of the tracking algorithm, only the movement of the nearer (i.e., right) foot was analyzed. No statistics are applied in the treadmill case since only single measurements are recorded (= 1 measurement per subject)

\section{Results}

\section{Studying transition under non-laboratory conditions}

Figure 3 presents the mean stride parameters for all 20 subjects tested outside the laboratory. Strides prior to transition are indicated with positive signs. Strides after transition are indicated with negative signs. The transition stride (stride 0 ) is the first stride with a single floating phase.

Mean stride velocities: Figure $3 \mathrm{~A}$ shows that the main increase in velocity occurred between TS and $-2 S(2.19 \pm 0.11 \mathrm{~m} / \mathrm{s}$ and $2.75 \pm 0.15$ $\mathrm{m} / \mathrm{s}$, respectively), where $-2 \mathrm{~S}$ refers to the stride which trails two strides behind TS. This corresponds to an acceleration of $0.62 \mathrm{~m} / \mathrm{s}^{2}$. Maximum deceleration in the RWT was registered between $3 \mathrm{~S}$ and $-1 \mathrm{~S}$ (and, respectively) at an acceleration of $-0.45 \mathrm{~m} / \mathrm{s}$ (Figure $3 \mathrm{~B}$ ). Stride velocities leveled off faster in the WRT than in the RWT, which indicates that the region in which the main transition happened comprised fewer strides in the WRT than in the RWT. In both transition regions, the main change in velocity occurred in the running pattern directly following or preceding TS.

In the absence of an agreed protocol for measuring transition speed outside the laboratory, we provide two sets of data that correspond to two different transition speed definitions:

1) Defined as the mean speed of TS, the transition speed is amounted to $2.18 \pm 0.11 \mathrm{~m} / \mathrm{s}$ for WRT and to $2.21 \pm 0.14 \mathrm{~m} / \mathrm{s}$ for RWT. These values are almost equal, and no hysteresis effect was observed.

2) Defined as the mean speed of the $1 S$ stride, the transition speed is amounted to $2.07 \pm 0.01 \mathrm{~m} / \mathrm{s}$ for WRT and $2.40 \pm 0.14 \mathrm{~m} / \mathrm{s}$ for RWT. Stride $1 S$ refers to the last walking stride in the WRT and to the last running stride in the RWT. In this case a transition hysteresis is observed. Clearly, the transition speed is very sensitive in a nonlaboratory setting and therefore it requires a precise measurement protocol for comparison to other studies.
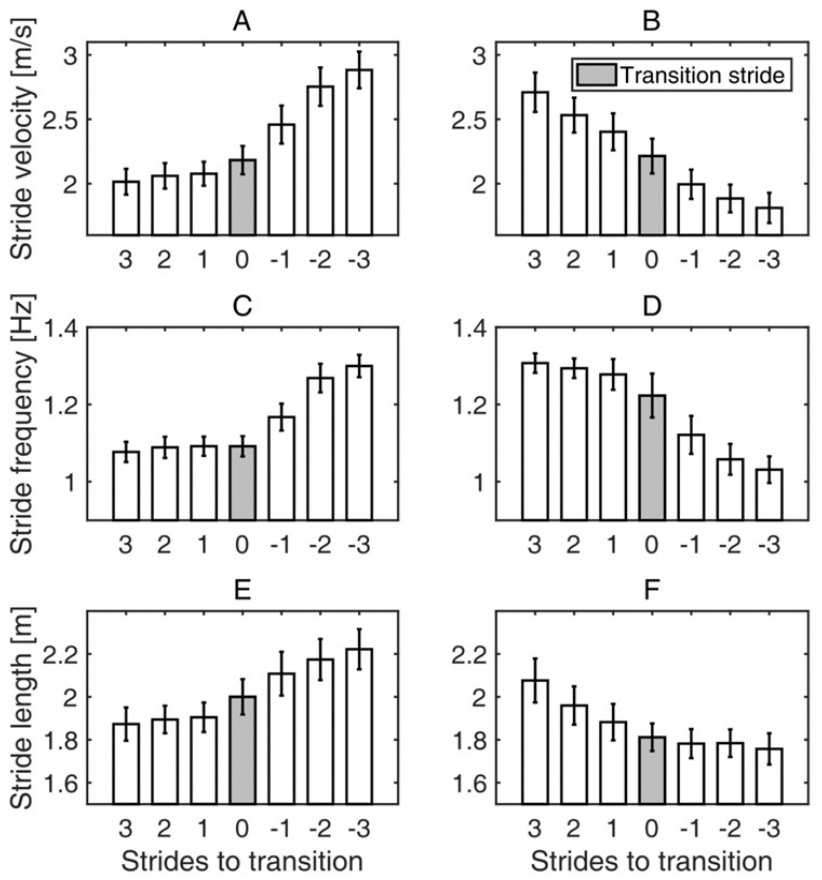

Figure 3: Stride velocity, stride frequency and stride length in the nonlaboratory case for seven strides centered around the transition stride. Figures $A, C$ and $E$ refer to the walk-run transition, and Figures $B, D$ and $F$ refer to the run-walk transition. 
Mean stride frequencies: The main frequency jump in the WRT happened within the first two strides in the running gait. It increased from $1.10 \pm 0.03 \mathrm{~Hz}$ (TS) to $1.27 \pm 0.04 \mathrm{~Hz}(-2 S)$. No significant frequency change could be detected before TS. During RWT, frequency was adapted almost symmetrically in both gaits with values of $1.28 \pm 0.04 \mathrm{~Hz}$ (1S) to $1.06 \pm 0.04 \mathrm{~Hz}(-2 \mathrm{~S})$ (Figure 3D). Outside these 2-4 strides, frequency changed hardly at all in the running gait and minimally in the walking gait. In general, standard deviations of stride frequency were relatively small compared to those of velocity and length. This indicates that stride frequency is the most robust parameter for defining the gait, as it is largely independent of a subject's individual style.

Mean stride lengths: The principal increase in stride length in the WRT was from $1.90 \pm 0.07 \mathrm{~m}(1 \mathrm{~S})$ to $2.17 \pm 0.10 \mathrm{~m}(-3 \mathrm{~S})$ (Figure 3E). During RWT, stride length decreased mainly in the running gait from $2.08 \pm 0.10 \mathrm{~m} \mathrm{(3S)}$ to $1.81 \pm 0.06 \mathrm{~m}$ (TS) (Figure 3F). The third stride $(-3 S)$ in the WRT was significantly longer $(2.22 \pm 0.09 \mathrm{~m})$ than the corresponding stride $(-3 \mathrm{~S})$ in the RWT $(2.08 \pm 0.1 \mathrm{~m})$, which indicates that stride lengths, like velocity, level off much faster in the WRT than in the RWT.

Individual Transition Behavior: Comparison between trained and untrained subjects: To determine whether gait shift is more regular for trained than for untrained subjects, we compared standard deviations for people who engage in physical activities regularly ( $\geq 2$ sport sessions per week (SSPW)) with those of people who exercise only occasionally or not at all $(<2 \mathrm{SSPW})$. Applying these criteria to subjects in Table 1, we obtained two equally large groups of 10 subjects each. For each group we applied Equations 8-11. Then we calculated the mean standard deviation from all strides. Our findings are presented in Table 2. We conclude that transition behavior does not differ between trained and untrained subjects, since the standard deviations are similar for both groups.

\section{Comparison of gait shifts on treadmill and outside the laboratory}

Figure 4 shows example stride parameters obtained for participant number 5 in the treadmill experiment. The following statements also apply to participants 6 and 11 (Figures 1 and 2).

Trivially, the measured stride velocity was identical to the predefined velocity profile of the moving belt at constant accelerations of $0.056 \mathrm{~m} / \mathrm{s}^{2}$ in the WRT and $0.056 \mathrm{~m} / \mathrm{s}^{2}$ in the RWT. These are much lower than the accelerations observed outside the laboratory. Further, the imposed velocity increments on the treadmill were uniform, whereas the velocity profile in the non-laboratory case was non-linear.

During walking, the stride frequency changed linearly with velocity. Analogously to the non-laboratory case (Figure 3C and D), frequency shifted abruptly to the attractor of the new gait. During running, when belt velocity was further increased, stride frequency

\begin{tabular}{|c|c|c|c|c|c|c|}
\hline & \multicolumn{3}{|c|}{ WRT } & \multicolumn{3}{|c|}{ RWT } \\
\hline & $\begin{array}{l}\sigma\left(v_{\mathrm{s}}\right) \\
{[\mathrm{m} / \mathrm{s}]}\end{array}$ & $\sigma\left(f_{s}\right)[H z]$ & $\sigma\left(I_{s}\right)[m]$ & $\begin{array}{c}\sigma\left(\mathrm{v}_{\mathrm{s}}\right) \\
{[\mathrm{m} / \mathrm{s}]}\end{array}$ & $\sigma\left(f_{s}\right)[H z]$ & $\sigma\left(I_{s}\right)[\mathrm{m}]$ \\
\hline SSPW $<=2$ & 0.116 & 0.029 & 0.083 & 0.138 & 0.041 & 0.078 \\
\hline SSPW $>2$ & 0.124 & 0.030 & 0.08 & 0.121 & 0.036 & 0.077 \\
\hline
\end{tabular}

Table 2: Comparison of the transition behaviors of subjects with regular sport session per week (SSPW $\geq 2$ ) to those who exercise only occasionally (SSPW <2). $(\sigma(p))$ is the mean standard deviation value of all seven strides within the transition region. $p$ is in place of the stride velocity $v_{s}$, the stride frequency $f_{s}$ or the stride length $\mathrm{I}_{\mathrm{s}}$. Each group comprised 10 subjects. It can be seen that the two groups show no significant differences in any of the stride parameters.
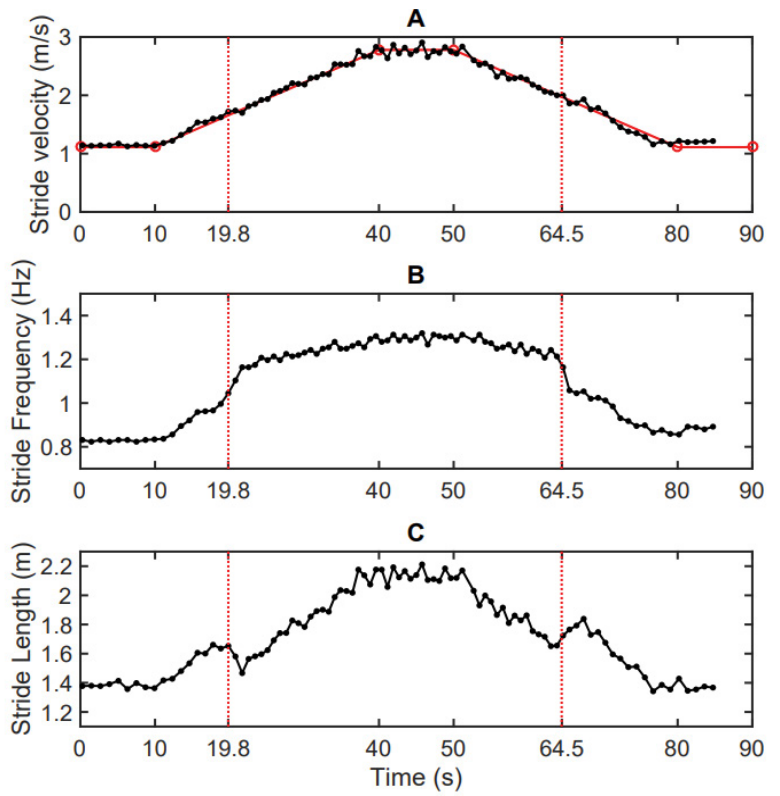

Figure 4: Example illustration of stride parameters from subject 11 measured on a treadmill. Vertical red lines mark the transition center. The data points (black dots) indicate stride parameters recorded for the right leg. The adjusted (red line in Figure A) and measured (black line in Figure A) velocity profiles are identical. During transition, frequency shifts abruptly towards the attractor of the new gait, whereas length shows an inversely proportional behaviour: lengths decrease in the WRT and increase in the RWT. Outside the transition area, stride frequency almost plateaus for the running gait, whereas stride length changes linearly with velocity.

remained largely unchanged. We observed that, in WRT outside the laboratory, stride frequency shifted mainly after the transition stride, whereas on a treadmill it shifted significantly before that stride for all 3 subjects tested. Stride lengths showed an inverted behavior during transition. Unlike in the non-laboratory case (Figure $3 \mathrm{E}$ and F), strides gradually decreased in length when the belt was sped up and increased when the belt was slowed down. Outside the transition regions, stride length changed linearly with speed.

\section{Discussion}

We investigated human gait transition based on three parameters: stride velocity, stride frequency and stride length. Twenty subjects were studied outside the laboratory and three of them additionally on a treadmill. Depending on the environment we used different techniques to study transition. For measurements outside the laboratory we developed a wearable device on the basis of IMUs and pressure sensors. On the treadmill, a video-based motion-capture system was used to track the movement of the lower limbs. All measurement data was transformed into a global frame (with Equations 2 and 12). Thus, treadmill parameters and parameters obtained in the non-laboratory environment could be meaningfully compared. Under non-laboratory conditions, we found that velocity varied non-linearly and at much higher accelerations (up to $0.62 \mathrm{~m} / \mathrm{s}^{2}$ ) than those imposed on a treadmill. This is in similar range as measured by Smet and colleagues $\left(0.7 \mathrm{~m} / \mathrm{s}^{2}\right)[5,6]$

Accelerations in the WRT were higher than those in the RWT (Figure 3A and B). Stride length leveled off faster in the WRT than in the RWT (Figure 3E and F). Both findings indicate that the WRT is faster-paced, within fewer strides than the RWT. The fast transition 
between gait patterns suggests that a particular velocity band is avoided. This can be explained as an efficiency measure of the body, where costs, in terms of energy expenditure per unit distance, are small for either walking at slower speeds or running at higher speeds, as proposed by [14]. The slower transition of the RWT could be an intuitive measure of the locomotive system to smoothly absorb kinetic energy and therefore protect the body from pain or injuries.

A few thoughts on the meaning of transition speed outside the laboratory context: Firstly, we saw that the stride-wise velocity increments were much higher than those on a treadmill (in our case by a factor of approximately 10). Secondly, individual transition speed can be measured more easily and more accurately on a treadmill. For instance, subjects can be asked whether, at a constant belt speed, walking or running is preferred [9]. This does not require knowledge of the exact time point at which the transition is executed, whereas under non-laboratory conditions the time point at which the transition is evaluated makes a great difference. We provided two definitions of the transition speed: (i) when equated to the velocity of the transition stride, the transition speeds of WRT and RWT were very similar (2.2 $\mathrm{m} / \mathrm{s}$ ) and, unlike in the treadmill case [15], a hysteresis effect could not be inferred. (ii) When the transition speed was defined as the velocity of the last walking and running strides of the WRT and the RWT, respectively, a hysteresis effect [4] was observed, since the values were $2.1 \mathrm{~m} / \mathrm{s}$ (WRT) and $2.4 \mathrm{~m} / \mathrm{s}$ (RWT). Comparisons of transition speeds obtained from different environments using different definitions must therefore be interpreted with care.

From Figure $3 \mathrm{C}$ and $\mathrm{D}$ we saw that stride frequency shifted abruptly and leveled off very fast, whereas stride velocity and stride length adapted slower. This shows that, compared to the other parameters, stride frequency adapts most rapidly to the new gait pattern. Looking at the standard deviations in Figure 3, we found that frequency varied less than velocity and length. This indicates that stride frequency adaptation is more similar between individuals than adaption of stride velocity and length.

In general, transition appeared to be strongly influenced by individual behavioral patterns. However, we found that engaging regularly in sports activities did not result in a steadier or more efficient transition (i.e., standard deviations of the stride parameters were not smaller) (Table 2). Therefore our results coincide well with the conclusion obtained in the work [16] that the PTS is independent from the training status. Whether this also applies to professional runners remains an open question.

Most importantly, we found that, stride frequency shifted similarly inside and outside the laboratory, whereas stride lengths showed exactly the opposite behavior. In WRT, when velocity was increased, stride lengths decreased. In RWT, when velocity was decreased, stride length increased. This can be explained as follows: Imposed accelerations were much smaller on the treadmill, but frequency was shifted almost identically in both environments. As velocity is constrained on a treadmill, the product of stride length times frequency is also constrained according to Equation 1. In conclusion, since frequency is preserved, stride length must decrease in order not to violate the velocity constraint. In a non-laboratory environment, subjects can choose frequency and length independently because velocity is a free parameter. In this case, we saw that lengths increased in the WRT and decreased in the RWT. This interplay between stride frequency and length clearly illustrates the differences in transition depending on the environment.

Stride frequency is a robust parameter which is largely unaffected by environmental changes. Due to its ability to adapt fast and its nearinvariance to individual behavioral patterns, this parameter can be used to accurately and reliably study gait. From a practical point of view, stride frequency has the decisive advantage over velocity and length that it can be measured without an IMU. In the simplest case, a single pressure sensor is sufficient to determine the parameter properly, and no sophisticated software algorithms are necessary.

\section{Acknowledgement}

We thank all the participants of the experiments. We thank DI in Anna Theresia Stadler and Dlin Roland Theresa for technical support and advices. We thank Dr. Ingrid Abfalter for serving as an editor.

\section{References}

1. Hu BH, Rouse EJ, Hargrove LJ (2017) Using bilateral lower limb kinematic and myoelectric signals to predict locomotor activities: A pilot study. IEEE Comp Soc pp: 98-101.

2. Alexander RM (1996) Walking and Running. The Mathematical Gazette 80: 262.

3. Hreljac A (1995) Effects of physical characteristics on the gait transition speed during human locomotion. Hum Mov Sci 14: 205-216.

4. Diedrich FJ, Warren WH Jr (1995) Why change gaits? Dynamics of the walkrun transition. J Exp Psychol Hum Percept Perform 21: 183-202.

5. De Smet K, Segers V, Lenoir M, De Clercq D (2009) Spatiotemporal characteristics of spontaneous overground walk-to-run transition. Gait Posture 29: 54-58.

6. De Smet K, Malcolm P, Lenoir M, Segers V, De Clercq D (2009) Effects of optic flow on spontaneous overground walk-to-run transition. Exp Brain Res 193. $501-508$

7. Kram R, Domingo A, Ferris DP (1997) Effect of reduced gravity on the preferred walk-run transition speed. J Exp Biol 200: 821-826.

8. Hreljac A (1993) Preferred and energetically optimal gait transition speeds in human locomotion. Med Sci Sports Exerc 25: 1158-1162.

9. Hreljac A, Imamura R, Escamilla RF, Edwards WB (2007) Effects of changing protocol, grade, and direction on the preferred gait transition speed during human locomotion. Gait Posture 25: 419-424.

10. Hansen EA, Kristensen LAR, Nielsen AM, Voigt M, Madeleine P (2017) The role of stride frequency for walk-to-run transition in humans. Sci Rep 7: 2010.

11. Bebek Ö, Suster MA, Rajgopal S (2010) Personal Navigation via HighResolution Gait-Corrected Inertial Measurement Units. IEEE Trans Instrum Meas 59: 3018-3027.

12. Tian X, Chen J, Han Y, Shang J, Li N (2016) A Novel Zero Velocity Interval Detection Algorithm for Self-Contained Pedestrian Navigation System with Inertial Sensors. Sensors (Basel) 16: 1578.

13. Fischer C, Talkad Sukumar $P$, Hazas M (2013) Tutorial: Implementing a Pedestrian Tracker Using Inertial Sensors. IEEE Pervasive Comput 12: 17-27.

14. Long LL, Srinivasan M (2013) Walking, running, and resting under time distance, and average speed constraints: optimality of walk-run-rest mixtures J R Soc Interface 10: 20120980.

15. Abdolvahab M, Carello C (2015) Functional distance in human gait transition. Acta Psychol (Amst) 161: 170-176.

16. Rotstein A, Inbar O, Berginsky T, Meckel Y (2005) Preferred transition speed between walking and running: effects of training status. Med Sci Sports Exerc 37: $1864-1870$. 